\title{
Modulation of hepatic mRNA-miRNA panel linked to NAFLD/NASH Hippo signaling and gut microbiota after administration of kefir in Non-Alcoholic Steatohepatitis Rat Model
}

Noha Salah

Ain Shams Faculty of Medicine

Marwa Matboli1

Ain Shams Faculty of Medicine

Amal Mansour

Ain Shams Faculty of Medicine

Nagwa M Abo El Magd

Ain Shams Faculty of Medicine

Amany Helmy Hasanin

Ain Shams Faculty of Medicine

Mohamed Kamel Hassan

Zewail City for Science \& Technology

Sanaa Eissa ( $\nabla$ drsanaa_mohamed@med.asu.edu.eg)

Ain Shams Faculty of Medicine

\section{Research Article}

Keywords: NASH, Hippo signaling, Probiotic, SOX11, AMOTL2, SMAD4, miRNA

Posted Date: December 6th, 2021

DOI: https://doi.org/10.21203/rs.3.rs-1138418/v1

License: (c) (1) This work is licensed under a Creative Commons Attribution 4.0 International License.

Read Full License 


\section{Abstract}

Non-alcoholic steatohepatitis (NASH) is the clinically aggressive variant of non-alcoholic fatty liver disease. Hippo pathway dysregulation can contribute to NASH development and progression. The use of probiotics is effective in NASH management. Our aim is to investigate the efficacy of kefir Milk in NASH management via modulation of hepatic mRNA-miRNA based panel linked to NAFLD/NASH Hippo signaling and gut microbita regulated genes which was identified using bioinformatics tools. Firstly, we analyzed mRNAs (SOX11, SMAD4 and AMOTL2), and their epigenetic regulator (miR-6807) followed by validation of target effector proteins (TGFB1, IL6 and HepPar1). Molecular, biochemical, and histopathological, analyses were used to evaluate the effects of kefir on high sucrose high fat (HSHF) diet -induced NASH in rats. We found that administration of Kefir proved to prevent steatosis and development of the inflammatory component of NASH. Moreover, Kefir improved liver function and lipid panel. At the molecular level, kefir down-regulated the expression of miR 6807-5p with subsequent increase in the expression of SOX 11, AMOTL2 associated with downregulated SMAD4, resulting in reduction in the expression of the inflammatory and fibrotic markers, IL 6 and TGF- $\beta 1$ in the treated and prophylactic groups compared to the untreated rats. In conclusion, Kefir suppressed NASH progression and improved both fibrosis and hepatic inflammation. The produced effect was correlated with modulation of SOX11, SMAD4 and AMOTL2 mRNAs) - (miR-6807-5p) - (TGFB, IL6 and, HepPar1) expression.

\section{Introduction}

Nonalcoholic fatty liver disease (NAFLD) is a pathological condition with accumulation of fat in the liver with no excessive alcohol intake, varying from simple hepatic steatosis (SS) to nonalcoholic steatohepatitis (NASH) with fibrosis and cirrhosis [1]. Due to the increasing epidemic of obesity and type 2 diabetes worldwide, a 178\% rise in liver deaths has been estimated because of NASH by 2030 [2]. NASH could be induced by multiple conditions acting in parallel, including genetic predisposition, oxidative stress, abnormal lipid metabolism, lipotoxicity, mitochondrial dysfunction, endoplasmic reticulum stress, altered production of cytokines and adipokines and gut dysbiosis. Accordingly, hepatic inflammation could precede steatosis in NASH [3]. At the present time, no FDA-approved medical treatment for NASH or liver fibrosis are confirmed [4].

Hippo signaling pathway was previously reported to have a critical role in the regulation of hepatic size, proliferation, apoptosis, and stress response[5]. Accordingly, its dysregulation could have an effect on NASH pathogenesis via liver dedifferentiation and tumorigenesis [6].

Moreover, former data have reported that noncoding RNAs (ncRNA), including microRNAs (miRNAs) and long noncoding RNAs (IncRNAs) [7] may play crucial regulatory roles in NASH initiation and progression. The coregulatory links between these ncRNAs may demonstrate the molecular regulation and its involvement in NASH progression and could be used as appropriate biomarkers for assessing severity of the disease [8]. 
Additionally, the use of intestinal microbiota as a potential therapeutic target for NASH have been achievable. Probiotics are live microorganisms, which give health benefits to the host if consumed in appropriate amounts [9]. Probiotics slow down disease progression and hinder gastrointestinal complications by affecting intestinal flora, intestinal permeability, and inflammatory response [10]. Currently, the use of probiotics in the management of NAFLD have not been addressed by any guidelines and the molecular mechanisms of their health benefits are not entirely known as well [11].

Kefir is an acidic-alcoholic fermented milk, produced by kefir grains. Therefore, it is stable and has a precise balance of lactic acid bacteria and yeast [12]. It contains around 30 unique species of "good bacteria" that could benefit gut health [13] and so used as a potential therapy to treat gastrointestinal diseases and ischemic heart disease [14] because of its microflora, and the existence of some metabolites as organic acids. Further, it has several biological effects e.g., antibacterial, antioxidant, immunomodulatory, antidiabetic, and cholesterol lowering actions [15]. Ho et al.21 reported that kefir milk has anti-adipogenic effects by suppressing adipocyte differentiation and inhibiting the expression of the SREBP-1, ACC and FAS proteins during cholesterol synthesis [16].

Clearly, bioinformatics presents novel clues and initial data for screening potential biomarkers for different diseases and drug monitoring [17]. Moreover, it can be used to identify genes related to specific biological functions and predict drug-molecular signaling that may lead to a breakthrough in targeted therapy [18].

Therefore, the present study aimed at investigating the efficacy of kefir Milk in NASH management via modulation of hepatic mRNA-miRNA based panel linked to NAFLD/NASH Hippo signaling and gut microbiota regulated genes which are identified using bioinformatics tools.

\section{Materials And Methods}

\subsection{Chemicals and drugs}

Cholesterol and cholic acid were purchased from Ralin BV (Lijinbaan, Netherlands). Ready-made kefir milk was purchased from Heal Pharmaceutical every week and stored at temperature $\left(4^{\circ} \mathrm{C}\right)$, it contains Lactobacillus lactis as the largest bacterial colony representing approximately $80 \%$ and Lactobacillus paracasei, Lactobacillus acidophilus, Lactobacillus delbrueckii ssp. bulgaricus, Lactobacillus Plantarum, Lactobacillus kefiranofaciens Leuconostoc subspecies., Streptococcus thermophilus and yeast of kefir (Danisco $\AA$ ) representing the other $20 \%$.

\subsection{Experimental animals and diets}

Forty male (6 weeks-old) Wistar rats (140-160 g) were obtained from the Scientific Research Institute, Cairo, Egypt, housed under specific conditions $\left(20 \pm 2^{\circ} \mathrm{C}\right), 12 \mathrm{~h}$ light/dark cycle with free access to water and normal rat chow. All experimental procedures were done according to the guidelines of the 
Institutional Animal Care and were approved by the Ethics Committee of Ain Shams Faculty of Medicine, Egypt (Ethical Approval Number; FWA000017585).

After a one-week acclimatization period, the rats were randomly divided into three groups $(n=8$ for each group where treatment was given orally by gavage: (I) Normal control group (NC) fed a normal pellet diet; (II) NASH model group, this group was further subdivided into lla: (9-weeks NASH model) and Ilb: (12weeks NASH model), Group III (probiotic-treated), this group was further subdivided into Illa: (NASH/early probiotic-treated), rats were fed HSFD for 12 weeks and received probiotic treatment daily from day one for 12 weeks and IIIb: (NASH/late probiotic-treated), rats were fed HSHF for 12 weeks and received probiotic treatment daily in the last 3 weeks. NASH model was induced by feeding rats high fat/high sucrose diet consisting of $70 \%$ standard chow, $20 \%$ lard, $10 \%$ sucrose, $1 \%$ cholesterol, and $0.25 \%$ cholic acid [19]. Kefir milk was mixed and then administered by oral gavage at a dose of $1.8 \mathrm{~mL} / \mathrm{rat} /$ day.All rats were weighed at the beginning of the study, at the end of each week, and before animal sacrifice (Figure 1).

Figure (1) Flow chart showing the experimental design of the animal studies. NASH: nonalcoholic steatohepatitis; HSHF: high sucrose and high fat.

\subsection{Blood sample and liver tissue collection}

At the end of the study, rats were fasted for $12 \mathrm{hrs}$ and anesthetized with one dose of urethane $(1.2 \mathrm{~g} / \mathrm{kg}$, IP) [25] for sacrificing. Blood samples were collected from retro-orbital vein, centrifuged at $3000 \mathrm{rpm}$ for $10 \mathrm{~min}$ for serum separation, then aliquoted and stored at $-20^{\circ} \mathrm{C}$ for subsequent biochemical analysis. Whole livers were removed promptly, weighed and dissected. Part of the hepatic tissues was immediately stored in $-80^{\circ} \mathrm{C}$ for RNAs and protein assessment whereas the remaining parts were rapidly fixed in $10 \%$ neutral buffered formalin for histopathological and immunohistochemical analyses.

\subsection{Assessment of liver function and lipid profile markers}

We assessed levels of serum aspartate transaminase (AST), alanine transaminase (ALT), total and direct bilirubin, gamma-glutamyl transferase (GGT), alkaline phosphatase (ALP) and Lipid profile [Total cholesterol (TC), Triglycerides (TG), HDL cholesterol (HDL-C), and LDL cholesterol (LDL-C)] in serum samples using commercial kits according to the manufacturer's instructions and proceeded by a multifunctional biochemistry analyzer (AU680, Beckman Coulter Inc, CA).

\subsection{Hepatic histopathological evaluation}

For light microscopy, the buffered formalin-fixed liver tissues were dehydrated in ethanol, immersed in paraffın wax and sliced into sections (5- $\mu \mathrm{m}$ thick). Standard hematoxylin-eosin (HE) for assessment of histological features of steatohepatitis and Masson's Trichrome staining for detecting collagen fibers were performed. Each section was scored for the severity of hepatic steatosis, inflammation and fibrosis individually according to the published criteria [20]. 
2.6 In-silico filtration of hepatic mRNA-miRNA based panel linked to hippo signaling, gut microbita regulated genes and NAFLD/NASH

Firstly; We have selected AMOTL2, SOX11 and SMAD4 mRNAs from public microarray dataset; QuickGO (https://www.ebi.ac.uk/QuickGO/), Comparative Toxicogenomics Database (http://ctdbase.org/) and Gene atlas database (https://www.ebi.ac.uk/gxa ) and by literature reviews because of their strong correlation to In-silico filtration of integrated mRNA-miRNA panel pathogenesis( Supplementary figure 1A1F). Secondly; we verified the gene ontology of the selected mRNAs and their link to Hippo signaling and hepatocyte stem cell proliferation/differentiation using Harmonizome database(http://amp.pharm.mssm.edu/Harmonizome/) ( Supplementary figure 2A-2E).. Thirdly; the selected mRNAs were verified for their relation to gut microbiota related genes by using Encyclopedia of gut microbiota regulated genes (http://microbiota.wall.gu.se/ ) (Supplementary figure 3A-3C). Fourthly; AMOTL2, SOX11 and SMAD4 mRNAs were then imported into Protein-Protein Interaction Networks Functional Enrichment Analysis( STRING) database(https://string-db.org/ ) to ensure their protein protein interaction and their linkage with HIPPO pathway target effectors( Supplementary figure 4A). Then, the mRNA was mapped to Hippo signaling using KEGG pathway of hippo (Supplementary figure 4B). Fifthly, we used miRWalk 3.0 (http://mirwalk.umm.uni-heidelberg.de/), that combine the prediction results of both TargetScan and MiRBase to retrieve has-miR-6807-5p based on target complementarity and ranking score (Supplementary figure $5 \mathrm{~A}-\mathrm{C}$ ). We verified the expression of has-miR-6807-5p in liver using miRmine database(Supplementary figure 6A). https://guanfiles.dcmb.med.umich.edu/mirmine/index.html). Lastly; pathway enrichment analysis of has-miR-6807-5p by using DIANA database tools: miRpath(http://snf515788.vm.okeanos.grnet.gr/) that confirmed its relation to Hippo signaling and stem cell proliferation/differentiation (Supplementary figure 6B).

\subsection{Extraction of total RNA (mRNA and miRNA)}

Total RNA from the liver tissue was extracted using miRNeasy Mini Kit (Cat. No. 217004, Qiagen, Germany) as per instructions from the manufacturer. The concentration and purity of total RNA were assessed using NanoDrop (Thermoscientific, USA); the purity of the isolated RNAs (A260/A280) was 1.82. The reverse transcription of the extracted total RNA into complementary DNA (cDNA) was immediately proceeded with miScript II RT (Cat. No. 218161, Qiagen) and RT2 First Strand Kit (Cat.No. 330404, Qiagen) according to the manufacturer's protocol using Thermo Hybaid PCR express (Thermo Fisher Scientific, Massachusetts, USA).

\subsection{Real-time quantitative polymerase chain reaction (RT- qPCR)}

The expression levels of SMAD4, SOX11 and AMOTL2 mRNAs ((Accession: NM_NM_005359.6, NM_ _003108.4 and NR_002819,_001113490.2, respectively) ) in the liver tissues were assessed using a Quantitect SYBR Green Master Mix Kit ROX qPCR Mastermix (Qiagen, Germany), hsa-miR-68075p(Accession: MIMAT0027514: 5' gugagccaguggaauggagagg 3') expression in tissue samples was assessed by using miScript SYBR Green PCR Kit (Cat. No. 218073, Qiagen, Germany). The GAPDH and 
Hs_SNORD72_11 miScript Primer were used as the house keeping gene to normalize the raw data and then compared with a control sample. RT-qPCR amplification was performed in an Applied Biosystems 7500 FAST Real Time PCR system (Applied Biosystems, Foster City, USA) thermal cycler. The PCR program for the SYBR Green-based qPCR was as follows: denaturation at $95^{\circ} \mathrm{C}$ for $15 \mathrm{~min} ; 40$ cycles of denaturation for $10 \mathrm{~s}$ at $94^{\circ} \mathrm{C}$; then annealing for $30 \mathrm{~s}$ at $55^{\circ} \mathrm{C}$; and lastly, extension for $30 \mathrm{~s}$ at $70^{\circ} \mathrm{C}$. Each reaction was performed in duplicate. The threshold cycle $(\mathrm{Ct})$ value of each sample was calculated using the StepOnePlus ${ }^{\text {Tm }}$ software v2.2.2 (Applied Biosystems). The $\mathrm{Ct}$ values over 36 were considered negative. The melting curves were analyzed to affirm the specificities of the amplicons for the SYBR Green-based PCR amplification. Calculation of the relative quantification of RNA expression was performed using the

Livak method, where $\mathrm{RQ}=2^{-\Delta \Delta C t}[21]$.

\subsection{Detection of Hepatocyte specific antigen (Hep Par 1) as Protein-based biomarkers for NAFLD/NASH progression (Garcia-Martinez et al.,2020) by immunohistochemical staining [22]}

For immunohistochemistry staining, 4- $\mu$ m thickness paraffin-embedded tissue sections were prepared and then incubated with primary Hep Par 1 antibody for 32 minutes at $37^{\circ} \mathrm{C}$ and stained using benchmark Ventana (GX) automated immunostainer.

The standard antigen retrieval method is Heat Induced Epitope Retrieval (HIER) in Tris-EDTA buffer pH 7.8 Cell Conditioning Solution (CC1) at $95^{\circ} \mathrm{C}$ for $44 \mathrm{~min}$ and is performed in the Ventana Discovery XT.

The staining protocol used was as follow: Loading slides, antibody and ultraView detection kit dispensers onto the BenchMark instrument. Selecting CC1 standard pretreatment. When the staining run is complete, the slides from the instrument are removed and rinsed well with wash buffer. Cover slip to prevent evaporation. The complex is then visualized with hydrogen peroxide substrate and 3,3 '-diaminobenzidine tetrahydrochloride (DAB) chromogen, which produces a brown precipitate that is readily observed by light microscopy.

The primary antibody used Hepatocyte specific antigen (Hep Par 1) (EP265), monoclonal rabbit antibody, 7 ml prediluted, dilution 1:100. Ref no: 264R-18, Lot no: 0000027465.

The result of immunohistochemistry staining was counted by using the method described by Khatun et al. 2003 [23] to calculate the histoscore (H-score) which involves a semiquantitative assessment of both the percentage of positive cells and the intensity of staining (graded as: 0 , non-staining; 1 , weak staining; 2, medium staining; and 3 , strong staining).

\subsection{Quantification of hepatic inflammatory cytokine IL-6 and fibrotic marker TGF- $\beta 1$ by Enzyme Linked Immunosorbant Assay (ELISA)}

Interleukin-6 (IL-6) and transforming growth factor $\beta 1$ (TGF- $\beta 1$ ) in liver tissues were determined using sandwich ELISA kits (cat no: E0079r and E0124R, respectively; EIAab, Wuhan, China) and according to manufacturer's instructions. IL- 6 and TGF- $\beta 1$ were selected as they are either highly involved in cell 
proliferation or strongly correlated to Hippo signaling pathway as well as strongly interacted with the preselected protein markers through string tool.

\subsection{Statistical Analysis}

The results were expressed as mean \pm SEM. Statistical analysis was carried out using GraphPad Prism, software program, version 6.0. Inc., CA, USA. Statistical difference among groups was determined using one-way ANOVA followed by post hoc test for comparison between more than two groups of parametric data. $P$ values $<0.05$ were considered statistically significant.

\section{Results}

\subsection{The effect of Kefir on body weight and relative liver weight}

There was a significant increase in the body weight of the rats fed the HSHF diet in NASH groups compared with rats fed with the normal pellet in normal controls $(\mathrm{NC}),(P<0.05)$. As well as Early Kefir group showed a significant decrease in body weight and the ratio of liver weight to body weight than NASH (12 weeks) group ( $P<0.05)$, Table 1.

Table (1): Body weight and liver mass in different study groups 


\begin{tabular}{|c|c|c|c|c|c|c|}
\hline & & $\begin{array}{l}\text { Control } \\
(n=8)\end{array}$ & $\begin{array}{l}\text { NASH } \\
\text { (9 weeks) } \\
(n=8)\end{array}$ & $\begin{array}{l}\text { NASH } \\
(12 \\
\text { weeks }) \\
(n=8)\end{array}$ & $\begin{array}{l}\text { Early Kefir } \\
\text { treatment } \\
(n=12)\end{array}$ & $\begin{array}{l}\text { Late Kefir } \\
\text { treatment } \\
(n=10)\end{array}$ \\
\hline & & Mean \pm SD & & & & \\
\hline $\begin{array}{l}\text { Body } \\
\text { weight (g) }\end{array}$ & Week 0 & $144.5 \pm 17$ & $\begin{array}{l}158.5 \pm \\
16.1\end{array}$ & $\begin{array}{l}153.3 \pm \\
6.79\end{array}$ & $161.2 \pm 17.37$ & $151.5 \pm 15.31$ \\
\hline & Week 9 & $240 \pm 32.95$ & $\begin{array}{l}288.8 \\
\pm 30.09 € \text { € }\end{array}$ & $\begin{array}{l}277.9 \pm \\
16.2^{€^{\star}}\end{array}$ & $\begin{array}{l}269.9 \pm 27.78 \\
€ a\end{array}$ & $301.7 \pm 18.79$ \\
\hline & Week12 & $263.8 \pm 41.4$ & & $\begin{array}{l}293.8 \pm \\
16.3\end{array}$ & $299.5 \pm 29.57$ & $312.7 \pm 12$ \\
\hline Liver weight & & $5.62 \pm 1.06$ & $\begin{array}{l}10.63 \\
\pm 1.99 *\end{array}$ & $\begin{array}{l}12.31 \pm \\
1.03^{\star}\end{array}$ & $10.67 \pm 1.23$ & $10.8 \pm 1.687$ \\
\hline $\begin{array}{l}\text { Values are } r \\
\text { Tukey's mul }\end{array}$ & $\begin{array}{l}n \pm S D ; n u \\
\text { e compari }\end{array}$ & $\begin{array}{l}\text { nber of anima } \\
\text { son test. Value }\end{array}$ & $\begin{array}{l}=8 \text { rats } / \text { ea } \\
\text { are mean } \pm\end{array}$ & $\begin{array}{l}\text { group. O } \\
\text { no. }=8 \text { ra }\end{array}$ & $\begin{array}{l}\text { Jay ANOVA test } \\
\text { ach group }\end{array}$ & lowed by \\
\hline * $P<0.001$ & mpared to & ontrol group. & & & & \\
\hline$\# P<0.001$ & mpared to & NASH (9 week & group. & & & \\
\hline$\delta P<0.001$ & mpared to & VASH (12 we€ & s) group. & & & \\
\hline a $P<0.001$ & mpared to & -ate Kefir. & & & & \\
\hline$€ P<0.05$ & & & & & & \\
\hline
\end{tabular}

The previous results were also accompanied by a significant increase in liver relative weight in NASH groups $(P<0.001)$ compared with the NC. Figure 2 . The liver to body weight ratio analysis indicated that both early and late Kefir treatment may be able to decrease liver injury, where they exhibited significant decrease in the liver/body weight ratios $(\mathrm{P}<0.001)$ compared to that of NASH group.

\subsection{Histopathological findings}

The liver from NC rats had a visible normal brownish red color smooth, and shiny appearance. On the contrary, NASH rats liver, showed the typical fatty liver features and looked enlarged, yellowish, and diffusely infiltrated with yellow spots. Additionally, a netlike pattern on the surface of livers from NASH group indicated the existence of fibrosis. Liver tissue of HSHF-fed rats administered with either early or late Kefir treatment appeared redder and lighter than those of NASH groups.

Under the light microscope (Figure 3), liver of NC rats showed normal histological structure without inflammation or fibrosis. It was formed of classic hepatic lobules which were nearly hexagonal in shape. On contrary, NASH groups demonstrated development of steatosis with large fat droplets, inflammation 
and fibrosis were visible as well (Figure 4). Compared with the NASH groups, the Kefir treated groups showed visible reductions in fat droplets, improvements in steatosis, and inflammation. The effects were more prominent in early treated group.

Figure (3\&4) The effect of early and late Kefir treatment on hepatic steatosis, inflammation and fibrosis using HE and Masson's trichrome staining (Magnifications: $\times 100$ ).(A) Control group showed no pathological changes, (B) NASH (9 weeks) group developed mainly micro vesicular steatosis, mild hepatic lobular inflammation and mild fibrous septa(star) (C) NASH (12 weeks) group showed diffuse and extensive micro and macro-vesicular steatosis, severe lobular inflammation, focal necrosis, hepatocellular ballooning, marked expansion of facultative hepatic progenitor cells (arrows), dense fibrous septa and collagen fibers (star) (D) Early Kefir and (E) Late Kefir groups showed a lower degree of steatosis, inflammation and fibrosis, where Early Kefir showed more remarkable histological improvements.

\subsection{The effect of Kefir treatment on liver function and lipid profile}

As illustrated in Table 2, compared to the NC group, the serum levels of AST, ALT, GGT, ALP, total bilirubin, and direct bilirubin have been increased significantly $(P<0.001)$ upon feeding the experimental rats with HSHF diet in NASH groups. On the other hand, oral administration of either early or late Kefir treatment, simultaneously with HSHF feeding, significantly reduced $(P<0.001)$ the elevated levels of these variables. Noticeably, this ameliorative effect was more pronounced when Kefir was administered prophylactically (Early Kefir).

Table (2): The effect of Kefir treatment on liver function and lipid profile 


\begin{tabular}{|c|c|c|c|c|c|}
\hline \multirow[t]{2}{*}{ Laboratory parameters } & Control & $\begin{array}{l}\text { NASH } \\
\text { (9 weeks) }\end{array}$ & $\begin{array}{l}\text { NASH } \\
\text { (12weeks) }\end{array}$ & Early Kefir & Late Kefir \\
\hline & \multicolumn{5}{|c|}{ Mean \pm SD } \\
\hline AST (U/L) & $\begin{array}{l}20.38 \\
\pm 3.5\end{array}$ & $71.1 \pm 4.6^{*}$ & $\begin{array}{l}80.5 \pm \\
5.451^{* \# €}\end{array}$ & $\begin{array}{l}33.67 \pm 5.48^{\#} \\
\delta \text { a }\end{array}$ & $60.8 \pm 4.367^{\# \delta}$ \\
\hline ALT (U/L) & $\begin{array}{l}27 \pm \\
4.5\end{array}$ & $81.88 \pm 3.94^{*}$ & $\begin{array}{l}126.6 \pm \\
20.03^{*} \#\end{array}$ & $\begin{array}{l}41 \pm 4.936^{\# \delta} \\
a\end{array}$ & $59.6 \pm 3.34^{\delta \#}$ \\
\hline GGT (U/L) & $\begin{array}{l}14.38 \\
\pm 2.2\end{array}$ & $64 \pm 7.03^{\star}$ & $\begin{array}{l}79.38 \\
\pm 13.39 \text { *\#€ }\end{array}$ & $\begin{array}{l}32.83 \pm 5.02^{\# \delta} \\
a €\end{array}$ & $43.3 \pm 9.62^{\delta \#}$ \\
\hline ALP (U/L) & $\begin{array}{l}29.13 \\
\pm 7.7\end{array}$ & $90.75 \pm 3.15^{\star}$ & $\begin{array}{l}105.9 \pm \\
7.2^{\star \#}\end{array}$ & $\begin{array}{l}51.5 \pm 6.11^{\#} \\
\delta a\end{array}$ & $68.4 \pm 7.57^{\# ~} \delta$ \\
\hline Total Bilirubin (mg/dL) & $\begin{array}{l}0.308 \\
\pm 0.05\end{array}$ & $1.06 \pm 0.17^{*}$ & $\begin{array}{l}1.844 \pm \\
0.505^{\star} \#\end{array}$ & $\begin{array}{l}0.463 \pm \\
0.05^{\#} \delta\end{array}$ & $0.73 \pm 0.067^{\delta \# €}$ \\
\hline Direct Bilirubin (mg/dL) & $\begin{array}{l}0.122 \\
\pm 0.02\end{array}$ & $0.57 \pm 0.08^{*}$ & $\begin{array}{l}0.806 \pm \\
0.14^{\star \#}\end{array}$ & $\begin{array}{l}0.223 \pm \\
0.05^{\#} \delta\end{array}$ & $\begin{array}{l}0.297 \pm 0.0194^{\#} \\
\delta\end{array}$ \\
\hline $\begin{array}{l}\text { TOTAL CHOLESTEROL } \\
(\mathrm{mg} / \mathrm{dL})\end{array}$ & $\begin{array}{l}88.25 \\
\pm 7.7\end{array}$ & $121.1 \pm 5.51^{*}$ & $\begin{array}{l}181.9 \pm \\
3.48^{\star \#}\end{array}$ & $\begin{array}{l}99 \pm 9.323^{\# \delta} \\
a\end{array}$ & $126.7 \pm 1.63^{\delta}$ \\
\hline Triglyceride (mg/dL) & $\begin{array}{l}45.38 \\
\pm 4.5\end{array}$ & $115.6 \pm 5.9^{*}$ & $\begin{array}{l}141.5 \pm \\
10.06^{\star \#}\end{array}$ & $73.5 \pm 3.7^{\# \varnothing \text { a }}$ & $94.5 \pm 4.882^{\# \delta}$ \\
\hline $\mathrm{HDL}-\mathrm{C}(\mathrm{mg} / \mathrm{dL})$ & $\begin{array}{l}64.50 \\
\pm 5.8\end{array}$ & $32 \pm 3.928^{*}$ & $\begin{array}{l}30.13 \pm \\
2.35^{*}\end{array}$ & $\begin{array}{l}50.17 \pm 3.04 \text { \# } \\
\delta a\end{array}$ & $60.5 \pm 2.321^{\# \delta}$ \\
\hline LDL-C (mg/dL) & $\begin{array}{l}14.68 \\
\pm 4\end{array}$ & $65.9 \pm 6.35^{*}$ & $\begin{array}{l}123.5 \pm \\
1.46^{*} \#\end{array}$ & $\begin{array}{l}34.13 \pm 11.3^{\#} \\
\delta \text { a }\end{array}$ & $47.3 \pm 4.187^{\# \delta}$ \\
\hline \multicolumn{6}{|c|}{$\begin{array}{l}\text { Values are mean } \pm S D \text {; number of animals }=8 \text { rats/each group. One-Way ANOVA test followed by } \\
\text { Tukey's multiple comparison test. LDL-C= low-density lipoproteins, } \mathrm{HDL}-\mathrm{C}=\text { High-density lipoprotein, } \\
\text { AST= Aspartate aminotransferase, } A L T=\text { Alanine aminotransferase, ALP= Alkaline phosphatase, } \\
\text { GGT= Gamma-glutamyl transferase. }\end{array}$} \\
\hline \multicolumn{6}{|c|}{ * $\mathrm{P}<0.001$ compared to control group. } \\
\hline \multicolumn{6}{|c|}{ \# P $<0.001$ compared to NASH (9 weeks) group. } \\
\hline \multicolumn{6}{|c|}{$\delta P<0.001$ compared to NASH (12 weeks) group. } \\
\hline \multicolumn{6}{|c|}{ a P $<0.001$ compared to Late Kefir. } \\
\hline$€ P<0.05$ & & & & & \\
\hline
\end{tabular}


Regarding lipid profile, there was a significant $(p<0.001)$ upsurge in the serum levels of TC, TG, and LDL$C$ coupled with a significant $(p<0.001)$ decrease of serum HDL-C level in animals of NASH groups compared to NC group. NASH (12 weeks) group exhibited statistically significant increase than NASH (9 weeks) group regarding TC, Triglyceride, LDL, Liver function tests and TGFB1 $(p<0.001)$.

When Kefir was given therapeutically along with HSHF diet, serum TC, TG and LDL-C levels decreased significantly $(P<0.001)$ while serum HDL-C increased significantly $(P<0.001)$, compared to NASH groups. These improving effects were enhanced by prophylactic supplementation with Kefir. The obtained results indicated that the use of gut microbiota-based treatments could diminish hepatocyte injury and improve serum lipid metabolism in NASH animal model.

\subsection{The effect of Kefir treatment on the expression of hepatic SOX11, SMAD4 and AMOTL2 mRNAs}

The expression level of SOX11, SMAD4 and AMOTL2 was assessed in the liver tissue of the experimental rats (Figure 5A-C). Results showed that feeding rats with HSHF diet caused a sharp significant $(\mathrm{P}<$ 0.001) decrease in SOX11 expression level in NASH group animals. The administration of Kefir has normalized the significant decreases in hepatic SOX11 expression level manifested in untreated NASH group animals. In addition, no significant change in the expression level of hepatic SOX11 was recorded between early and late treated groups. Meanwhile, NASH group recorded a sharp significant increase in level of SMAD4(P < 0.001), compared to NC which was significantly corrected by administration with Kefir treatment, compared to NASH group animals. Moreover, data showed that hepatic AMOTL2 expression levels in NASH group animals was significantly decreased $(P<0.001)$, compared to NC and was significantly corrected by administration with Kefir.

Figure (5) Effect of Kefir on the expression level of hepatic m-RNAs SOX11, SMAD4 and AMOTL2(A-C), hepatic miR 6807-5p (D). Values are mean \pm SEM; number of animals $=8$ rats/each group. * $P<0.001$ compared to control group, \# $\mathrm{P}<0.001$ compared to NASH (9 weeks) group, $\delta \mathrm{P}<0.001$ compared to NASH (12 weeks) group. One-way ANOVA followed by Tukey's multiple comparison test. $R Q=$ relative quantification, SOX11= SRY-RELATED HMG-BOX GENE 11, SMAD4= SMAD Family Member 4, AMOTL2=Angiomotin-like 2, hsa-miR 6807-5p= Homo sapiens miR 6807-5p.

\subsection{The effect of Kefir treatment on the expression of hepatic miR-6807-5p}

As shown in Figure 5D, the RQ of miR-6807-5p in the liver tissue of NASH group animals was drastically increased $(P<0.001)$ compared to NC. Furthermore, both early and late Kefir treatment normalized the expression of hepatic miR-6807-5p, compared with that of NASH groups.

\subsection{The effect of Kefir on Hepatic cell differentiation and proliferation}


The state of liver progenitor cell proliferation and differentiation were evaluated in the liver by immunohistochemical analysis and assessment of Hepatocyte specific antigen (HepPar1). The increased in HepPar1 expression seen in NC (Figure 6) compared to NASH groups results from Granular cytoplasmic staining due to mitochondrial binding. Hepatic progenitor cells (HPCs) gradually lose their biliary features, including markers as (HepPar1) and these transitional cells in the hepatocytic lineage become negative for the marker (Figure 6B\& C). Kefir administration showed up-regulation of HepPar1positive cells in the liver tissues and the effect was more prominent in group received early treatment.

Figure (6) Immunohistochemical staining for hepatocyte specific antigen (HepPar1). (A) Controls showed a strong signal with Hep Par 1 (hepatocyte marker) producing distinct granular, cytoplasmic staining of hepatocytes, whereas in both (B) NASH (9 weeks) and (C) NASH (12 weeks) groups were mostly negative with HepPar-1. By comparison, (96\%) hepatocytes in (D) Early Kefir group were immunoreactive for HepPar-1 and (E) Late Kefir group tended to show weaker, patchy positivity (HepPar1, $\times 100)$ indicated to hepatocyte differentiation.

\subsection{The effect of Kefir on hepatic level of IL- 6 and TGF- $\beta 1$}

The hepatic contents of IL- 6 and TGF- $\beta 1$ proteins were used as markers of inflammation and fibrogenesis, respectively. In the NASH groups, IL-6 and TGF- $\beta 1$ proteins were significantly increased ( $P<$ 0.001 , Figure 7), compared to NC group. Kefir administration significantly decreased TGF- $\beta 1$ compared with NASH groups $(P<0.001)$. These findings implied that both of early and late Kefir treatment effectively diminished the level of inflammation and fibrosis observed in NASH rats.

Figure (7) The effect of Kefir treatment on (A) Level of IL-6 (B) Level of TGF- $\beta 1$ in liver tissues from various groups. Values are mean $\pm S E M$, number of animals $=8$ rats/each group. ${ }^{*} P<0.001$ compared to control group, \# $\mathrm{P}<0.001$ compared to NASH (9 weeks) group, $\delta \mathrm{P}<0.001$ compared to NASH (12 weeks) group, $€ \mathrm{P}<0.05$. One-way ANOVA followed by Tukey's multiple comparison test. IL6= Interleukin 6, TGFB1 $=$ transforming growth factor beta 1 .

\section{Discussion}

Non-alcoholic fatty liver disease (NAFLD) has become a very common disease because of the prevailing increase in obesity worldwide. Currently, several NAFLD therapies are being targeted to improve insulin resistance (IR), but there is no effective treatment [24]. Probiotic treatment was shown to improve NASH through modulating insulin resistance, the key factor which plays a major role in the development of a serious liver condition [25]. Due to correlation between small intestinal bacterial overgrowth (SIBO) and NAFLD observed in experimental and clinical studies [26], [27], probiotics could also delay disease progression and prevent complications by modulating intestinal flora, intestinal permeability, and inflammatory response [28].

In this context, a high fat/high sucrose diet (HFD) induced NASH model experiment was conducted to assess the beneficial effects of the Kefir formula on the degree of hepatic fibrosis and steatosis, 
inflammation, and body composition via modulation at both epigenetic and genetic level. Previous studies have showed that kefir improved NAFLD regarding to BW, energy expenditure and basal metabolic rate through inhibition of the lipogenesis pathway [29]. This may explain our findings where the supplementation with Kefir prophylactically or therapeutically along with HFD, decreased the percentage and stage of steatosis, inflammation and fibrosis seen in NASH rats. Moreover, the serum levels of ALT and AST, and the hepatic content of fat, TGFB 1 and IL 6 proteins were significantly reduced with the treatment of the Kefir formula compared to NASH groups. In agreement with the previous finding that Kefir can diminish the inflammatory response in association with a decrease in tumor necrosis factor alpha (TNF-a), interleukin-1 beta (IL-1 $\beta$ ) and transforming growth factor beta (TGF- $\beta$ ) cytokines [30].

Hepatic stem/progenitor cells (HPCs or HpSCs, in humans) or oval cells (in rodents) are bipotential stem cells that can differentiate into mature hepatocytes and cholangiocytes [31]. They represent a reserve compartment that can be activated to reactive ductulus (Or ductular reaction: DR) only when the mature epithelial cells of the liver are continuously damaged or blocked in their replication or in cases of serious cell loss [32].

Hippo pathway could promote cell death and differentiation and inhibit cell proliferation, however, the regulatory mechanisms for this signaling pathway are not clearly understood [33]. The downstream effector YAP1 is shown to be involved in hepatic cell proliferation, survival, development and differentiation [34]. Activated YAP1 leads to activation of hepatic stellate cells (HSC), prolonged activation of these cells causes liver fibrogenesis [35]. Therefore, a key objective is to understand the mechanisms that stimulate the switch of quiescent HSCs in a healthy liver to activated, myofibroblastic HSCs in NASH.

In this sense, variety of public microarray databases and computation algorithms have been investigated in the current work, for the selection of hepatic mRNA-miRNA panel linked to NAFLD/NASH Hippo signaling pathway and gut microbita regulated genes. We identified 3 mRNAs (SOX11, SMAD4 and AMOTL2), their epigenetic regulator (miR-6807) and their target effector proteins (TGFB, IL6 and HepPar1). Protein -protein interaction between the selected mRNAs protein products and HIPPO target effectors was obvious by STRING database as shown in (supplementary figure 4A).

Cytosolic AMOTL2 proteins can attach YAP1 and WWTR1 (TAZ) in their unphosphorylated states, providing a Hippo-independent mechanism to down-regulate the activities of these proteins [36]. AMOTL2 mRNA is downregulated in the current study in the rat liver tissue of NASH compared to NC or treated groups $(P<0.001)$, which indicated the freeing of YAP1 with subsequent activation of hepatic stellate cells and fibrogenesis participating in the pathogenesis of NASH. On the other hand, AMOTL2 is up regulated by the intake of kefir in rats which possibly ameliorates the features of NASH in the treated rat group by bounding YAP1.

SMAD 4 interacts with SMAD2/3 and participates in the intracellular TGF- $\beta$ signaling pathway. Knockout of SMAD4 from mesangial cells resulted in inhibition of TGF- $\beta 1$-produced ECM synthesis [37].These previous published results confirmed that SMAD4 has a significant role in the pathogenesis of fibrosis by 
controlling the ability of SMAD3 to activate transcription of a number of fibrogenic genes (collagens), markers (a-SMA and E-cadherin) [38] and explain the significant overexpression of SMAD4 in NASH group than NC and treated groups observed in the current study $(P<0.001)$.

SOX-11(SRY-related HMG-box) is a member of the group C SOX transcription factor family involved in the regulation of embryonic development and in the determination of the cell fate [39]. Its mRNA shows decreased expression in NASH versus other study groups conducted in the current study $(P<0.001)$. This agrees with a previous study that validated hepatic expression of a subset of mRNAs and reported downregulation of SOX11 in Obesity-Related Nonalcoholic Steatohepatitis relative to normal tissue [40].

MiRNAs are reported to play important role in mediating the development of diseases, including NASH [5]. MiR 6807-5p is shown to be a novel microRNA biomarker for detecting gastric cancer (GC) but no previous reports have correlated its expression with the incidence or progression of NASH or with other liver diseases [41]. Our bioinformatics data analysis revealed its expression in liver (supplementary figure 6A) and its correlation to Hippo signaling pathway (supplementary.figure 6B). Moreover, SOX11, SMAD4 and AMOTL2 are shown to be direct mRNA targets of miRNA-6807-5p as was retrieved from miRwalk database.(supplementary figure $5 A-C$ ). Our results revealed that there was a significant increase in miR 6807-5p concomitant with marked increase in SMAD4 and significant down regulation of SOX11, and AMOTL2 mRNA levels in the liver tissue of NASH group compared to NC or treated groups. This deregulated molecular profile reverts to normal after treatment with kefir milk.

TGF- $\beta$ was significantly increased in NASH as compared with other groups $(P<0.001)$ which is explained by previous work reporting that activation of TGF- $\beta$ signaling pathway was involved in the occurrence and development of NASH through motivating HSCs and the formation of extracellular matrix (ECM) [42], [43].

Continuous increased triacylglycerols buildup produces reactive oxygen species (ROS) and proinflammatory cytokines e.g IL6, which induces NASH. IL-6 expression was significantly increased in the hepatic biopsy of patients with nonalcoholic steatohepatitis (NASH) than patients having simple steatosis or normal biopsies. Morever, there was a positive correlation between fibrosis staging and inflamation degree confirming the presence of hepatic IL-6 expression in human NASH [44]. Our results revealed a marked increase in IL- 6 level in rat NASH liver when compared to $N C(P<0.001)$ but it was returned back to normal levels after kefir treatment.

In the present study, proliferation of oval cells was enhanced in NASH groups and decreased in Kefir groups. Previous studies found that HPC activation and the expansion of DR have been correlated with progressive fibrosis in adult and pediatric NASH and in HCV associated cirrhosis [45].

In addition, upon differentiation towards hepatocytes, HPCs were previously shown to gradually lose their biliary features, including markers such as keratin 19 (K19) and keratin 7 (K7) [46] as well as Hepatocyte Paraffin 1(Hep Par1). For the best of our knowledge, Hep Par1 was used for the first time as a marker of 
hepatocellular differentiation in NASH where it is shown to be mostly negative in the liver of NASH group while $96 \%$ of hepatocytes were immunoreactive for HepPar-1. with kefir treatment (figure 6).

Taken all together, our experimental model hypothesized that treatment with probiotic Kefir downregulated miR 6807-5p with subsequent upregulation of of SOX 11, and AMOTL2 and downregulation of SMAD4. Accordingly, YAP1 is negatively regulated with its subsequent sequestration in the cytosol. This cytoplasmic translocation of YAP1 inhibited cell proliferation and suppressed the expression of inflammatory IL6 and fibrotic TGF- $\beta 1$ (figure 8).

\section{Conclusion}

kefir Milk was effective to improve the pathological and biochemical disturbances induced in NASH animal model through modulation of Hippo signaling/gut regulated genes linked RNA panel. This treatment down-regulated the expression of miR 6807-5p and SMAD4 as well as up-regulated the expression of SOX11\& AMOTL2 mRNAs in rat hepatic tissue. The protein products of these genes interact with YAP1 in the Hippo signaling pathway controlling inflammation, and fibrosis progression in NASH.

\section{Declarations}

\section{Author Contributions:}

Data curation, Noha Salah and Marwa Matboli; Formal analysis, Noha Salah and Marwa Matboli; Funding acquisition, Sanaa Eissa; Methodology, Noha Salah, Marwa Matboli, Nagwa Mahmoud, Amany Hasanin and Mohamed Hassan; Project administration, Sanaa Eissa; Resources, Marwa Matboli; Software, Marwa Matboli; Supervision, Amal Mansour and Sanaa Eissa; Validation, Amal Mansour; Visualization, Mohamed Hassan; Writing - original draft, Noha Salah, Marwa Matboli, Amal Mansour, Nagwa Mahmoud, Amany Hasanin and Mohamed Hassan; Writing - review \& editing, Sanaa Eissa.

Funding: The work was funded by Egyptian Academy of Scientific Research and Technology, JESOR grant ID: 2017-2961.

Institutional Review Board Statement: The study was approved by the ASU-Faculty of Medicine ethical committee (Ethical Committee Approval No FWA000017585).

Conflicts of Interest: The authors report no declarations of interest. The funders had no role in the design of the study; in the collection, analyses, or interpretation of data; in the writing of the manuscript, or in the decision to publish the results.

\section{References}

1. Siddiqui, M. S., Van Natta, M. L., Connelly, M. A., Vuppalanchi, R., Neuschwander-Tetri, B. A., Tonascia, J., \& Chalasani, N. Impact of obeticholic acid on the lipoprotein profile in patients with non-alcoholic 
steatohepatitis. Journal of Hepatology (2020), 72(1), 25-33.

2. Estes, C., Razavi, H., Loomba, R., Younossi, Z., \& Sanyal, A. J. Modeling the epidemic of nonalcoholic fatty liver disease demonstrates an exponential increase in burden of disease. Hepatology (2018), 67(1), 123-133.

3. Kutlu, O., Kaleli, H. N., \& Ozer, E. Molecular pathogenesis of nonalcoholic steatohepatitis-(NASH-) related hepatocellular carcinoma. Canadian Journal of Gastroenterology and Hepatology (2018), 2018.

4. EASL, E. EASO. Clinical Practice Guidelines for the Management of Non-Alcoholic Fatty Liver Disease, Obes Facts (2016), 9, 65-90.

5. Gadallah, S. H., Eissa, S., Ghanem, H. M., Ahmed, E. K., Hasanin, A. H., El Mahdy, M. M., \& Matboli, M. Probiotic-prebiotic-synbiotic modulation of (YAP1, LATS1 and NF2 mRNAs/miR-1205/IncRNA SRD5A3-AS1) panel in NASH animal model. Biomedicine \& Pharmacotherapy (2021), 140, 111781.

6. Ardestani, A., Lupse, B., \& Maedler, K. Hippo signaling: key emerging pathway in cellular and wholebody metabolism. Trends in Endocrinology \& Metabolism (2018), 29(7), 492-509.

7. Zhang, Z., Wen, H., Peng, B., Weng, J., \& Zeng, F. Downregulated Microrna-129-5p by Long NonCoding RNA NEAT1 Upregulates PEG3 Expression to Aggravate Non-Alcoholic Steatohepatitis. Frontiers in Genetics (2021), 11, 1407.

8. Huang R, Duan X, Fan J, Li G, Wang B. Role of noncoding RNA in development of nonalcoholic fatty liver disease. BioMed research international. 2019 Feb 26;2019.

9. Lavekar, A. S., Raje, D. V., Manohar, T., \& Lavekar, A. A. Role of probiotics in the treatment of nonalcoholic fatty liver disease: a meta-analysis. Euroasian journal of hepato-gastroenterology (2017), 7(2), 130.

10. Suez, J., Zmora, N., Zilberman-Schapira, G., Mor, U., Dori-Bachash, M., Bashiardes, S., \& Horn, M. Postantibiotic gut mucosal microbiome reconstitution is impaired by probiotics and improved by autologous FMT. Cell (2018), 174(6), 1406-1423.

11. Nobili, V., Putignani, L., Mosca, A., Del Chierico, F., Vernocchi, P., Alisi, A., \& Drago, L. Bifidobacteria and lactobacilli in the gut microbiome of children with non-alcoholic fatty liver disease: which strains act as health players?. Archives of medical science: AMS (2018), 14(1), 81.

12. Wang, S. Y., Chen, K. N., Lo, Y. M., Chiang, M. L., Chen, H. C., Liu, J. R., \& Chen, M. J. Investigation of microorganisms involved in biosynthesis of the kefir grain. Food microbiology (2012), 32(2), 274285.

13. Prado, M. R., Blandón, L. M., Vandenberghe, L. P., Rodrigues, C., Castro, G. R., Thomaz-Soccol, V., \& Soccol, C. R. Milk kefir: composition, microbial cultures, biological activities, and related products. Frontiers in microbiology (2015), 6, 1177.

14. Farnworth, Edward R., and Isabelle Mainville. "Kefir: a fermented milk product." Handbook of fermented functional foods 2 (2003): 89-127.

15. Vujičić, I. F., Vulić, M., \& Könyves, T. Assimilation of cholesterol in milk by kefir cultures. Biotechnology Letters (1992), 14(9), 847-850. 
16. Ho, J. N., Choi, J. W., Lim, W. C., Kim, M. K., Lee, I. Y., \& Cho, H. Y. Kefir inhibits 3T3-L1 adipocyte differentiation through down-regulation of adipogenic transcription factor expression. Journal of the Science of Food and Agriculture (2013), 93(3), 485-490.

17. Ye, Z., Wang, F., Yan, F., Wang, L., Li, B., Liu, T., \& Fu, Z. Bioinformatic identification of candidate biomarkers and related transcription factors in nasopharyngeal carcinoma. World journal of surgical oncology (2019), 17(1), 1-10.

18. Matboli, M., Gadallah, S. H., Rashed, W. M., Hasanin, A. H., Essawy, N., Ghanem, H. M., \& Eissa, S. mRNA-miRNA-IncRNA Regulatory Network in Nonalcoholic Fatty Liver Disease. International journal of molecular sciences (2021), 22(13), 6770.

19. Guo, J. H., Han, D. W., Li, X. Q., Zhang, Y., \& Zhao, Y. C. The impact of small doses of LPS on NASH in high sucrose and high fat diet induced rats. Eur Rev Med Pharmacol Sci (2014), 18(18), 2742-2747.

20. Schnabl, B., Farshchi-Heydari, S., Loomba, R., Mattrey, R. F., Hoh, C. K., Sirlin, C. B. \& Vera, D. R. Staging of fibrosis in experimental non-alcoholic steatohepatitis by quantitative molecular imaging in rat models. Nuclear medicine and biology (2016), 43(2), 179-187.

21. Livak KJ \& Schmittgen TD: Analysis of relative gene expression data using realtime quantitative PCR and the 2(Delta Delta C(T)) Method. Methods (2001);25(4):402-8.

22. Garcia-Martinez, I., Alen, R., Rada, P., \& Valverde, A. M. Insights into extracellular vesicles as biomarker of NAFLD pathogenesis. Frontiers in Medicine (2020), 7, 395.

23. Khatun, S., Fujimoto, J., Toyoki, H., \& Tamaya, T. Clinical implications of expression of ETS-1 in relation to angiogenesis in ovarian cancers. Cancer science (2003), 94(9), 769-773.

24. Smeuninx, B., Boslem, E., \& Febbraio, M. A. Current and future treatments in the fight against nonalcoholic fatty liver disease. Cancers (2020), 12(7), 1714.

25. Silva-Sperb, A. S., Moraes, H. A., de Moura, B. C., Alves, B. C., Bruch-Bertani, J. P., Azevedo, V. Z., \& Dall'Alba, V. Effect of probiotic supplementation in nonalcoholic steatohepatitis patients: PROBILIVER TRIAL protocol. Trials (2019), 20(1), 1-8.

26. Takaki, A., Kawai, D., \& Yamamoto, K. Multiple hits, including oxidative stress, as pathogenesis and treatment target in non-alcoholic steatohepatitis (NASH). International journal of molecular sciences (2013), 14(10), 20704-20728.

27. Caligiuri, A., Gentilini, A., \& Marra, F. Molecular pathogenesis of NASH. International journal of molecular sciences (2016), $17(9), 1575$.

28. Feldman, M., Friedman, L. S., \& Brandt, L. J. (Eds.). Sleisenger and Fordtran's gastrointestinal and liver disease E-book: pathophysiology, diagnosis, management (2020),(Vol. 1). Elsevier.

29. Chen, H. L., Tsai, T. C., Tsai, Y. C., Liao, J. W., Yen, C. C., \& Chen, C. M. Kefir peptides prevent highfructose corn syrup-induced non-alcoholic fatty liver disease in a murine model by modulation of inflammation and the JAK2 signaling pathway. Nutrition \& diabetes (2016), 6(12), e237-e237.

30. Peluzio MD, e Dias MD, Martinez JA, Milagro FI. Kefir and intestinal microbiota modulation: implications in human health. Frontiers in Nutrition. (2021);8. 
31. Beaufrère A, Calderaro J, Paradis V. Combined hepatocellular-cholangiocarcinoma: an update. Journal of Hepatology. (2021) Feb 3.

32. Van Haele, M., \& Roskams, T. Hepatic progenitor cells: an update. Gastroenterology Clinics (2017), 46(2), 409-420.

33. Meng, Z., Moroishi, T., \& Guan, K. L. Mechanisms of Hippo pathway regulation. Genes \& development (2016), 30(1), 1-17.

34. Manmadhan, S., \& Ehmer, U. Hippo signaling in the liver-a long and ever-expanding story. Frontiers in cell and developmental biology (2019), 7, 33.

35. Yu, H. X., Yao, Y., Bu, F. T., Chen, Y., Wu, Y. T., Yang, Y. \& Meng, X. M. Blockade of YAP alleviates hepatic fibrosis through accelerating apoptosis and reversion of activated hepatic stellate cells. Molecular immunology (2019), 107, 29-40.

36. Rojek, K. O., Krzemień, J., Doleżyczek, H., Boguszewski, P. M., Kaczmarek, L., Konopka, W., \& Prószyński, T. J. Amot and Yap1 regulate neuronal dendritic tree complexity and locomotor coordination in mice. PLoS biology (2019), 17(5), e3000253.

37. Tsuchida KI, Zhu Y, Siva S, Dunn SR, Sharma K. Role of Smad4 on TGF- $\beta$-induced extracellular matrix stimulation in mesangial cells. Kidney international. 2003 Jun 1;63(6):2000-9.

38. Xu F, Liu C, Zhou D, Zhang L. TGF- $\beta$ /SMAD pathway and its regulation in hepatic fibrosis. Journal of Histochemistry \& Cytochemistry. 2016 Mar;64(3):157-67.

39. Jiao, Y., Zhao, J., Zhang, Z., Li, M., Yu, X., Yang, Y. \& Li, X. SRY-Box containing Gene 4 promotes liver steatosis by upregulation of SREBP-1c. Diabetes (2018), 67(11), 2227-2238.

40. Gerhard, G. S., Legendre, C., Still, C. D., Chu, X., Petrick, A., \& DiStefano, J. K. Transcriptomic profiling of obesity-related nonalcoholic steatohepatitis reveals a core set of fibrosis-specific genes. Journal of the Endocrine Society (2018), 2(7), 710-726.

41. Iwasaki, H., Shimura, T., Yamada, T., Okuda, Y., Natsume, M., Kitagawa, M.,\& Kataoka, H. A novel urinary microRNA biomarker panel for detecting gastric cancer. Journal of gastroenterology (2019), 54(12), 1061-1069.

42. Richardson, N., Ng, S. T., \& Wraith, D. C. Antigen-specific immunotherapy for treatment of autoimmune liver diseases. Frontiers in immunology (2020), 11, 1586.

43. Qin, G., Wang, G. Z., Guo, D. D., Bai, R. X., Wang, M., \& Du, S. Y. Deletion of Smad4 reduces hepatic inflammation and fibrogenesis during nonalcoholic steatohepatitis progression. Journal of digestive diseases (2018), 19(5), 301-313

44. Wieckowska A, Papouchado BG, Li Z, Lopez R, Zein NN, Feldstein AE. Increased hepatic and circulating interleukin-6 levels in human nonalcoholic steatohepatitis. Official journal of the American College of Gastroenterology| ACG. 2008 Jun 1;103(6):1372-9.

45. Nobili, V., Carpino, G., Alisi, A., Franchitto, A., Alpini, G., De Vito, R.,\& Gaudio, E. Hepatic progenitor cells activation, fibrosis, and adipokines production in pediatric nonalcoholic fatty liver disease. Hepatology (2012), 56(6), 2142-2153. 
46. Ko, S., Russell, J. O., Molina, L. M., \& Monga, S. P. Liver progenitors and adult cell plasticity in hepatic injury and repair: knowns and unknowns. Annual Review of Pathology: Mechanisms of Disease (2020), 15, 23-50.

47. Medrano,M.,Pérez,P.F.,and Abraham,A.G. Kefir anantagonizes cytopathiceffectsof Bacilluscereus extracellularfactors. Int.J.FoodMicrobiol.(2008). 122, 1-7.doi:10.1016/j.ijfoodmicro.2007.11.046

\section{Figures}

\section{Animal study design}

Control

NASH $(9$

weeks)

NASH $(12$ weeks)

Early Kefir

\section{Late Kefir}

- Normal pellet diet (12 weeks)

- HSHF diet (9 weeks)

\section{Figure 1}

Flow chart showing the experimental design of the animal studies. NASH: nonalcoholic steatohepatitis; HSHF: high sucrose and high fat. 


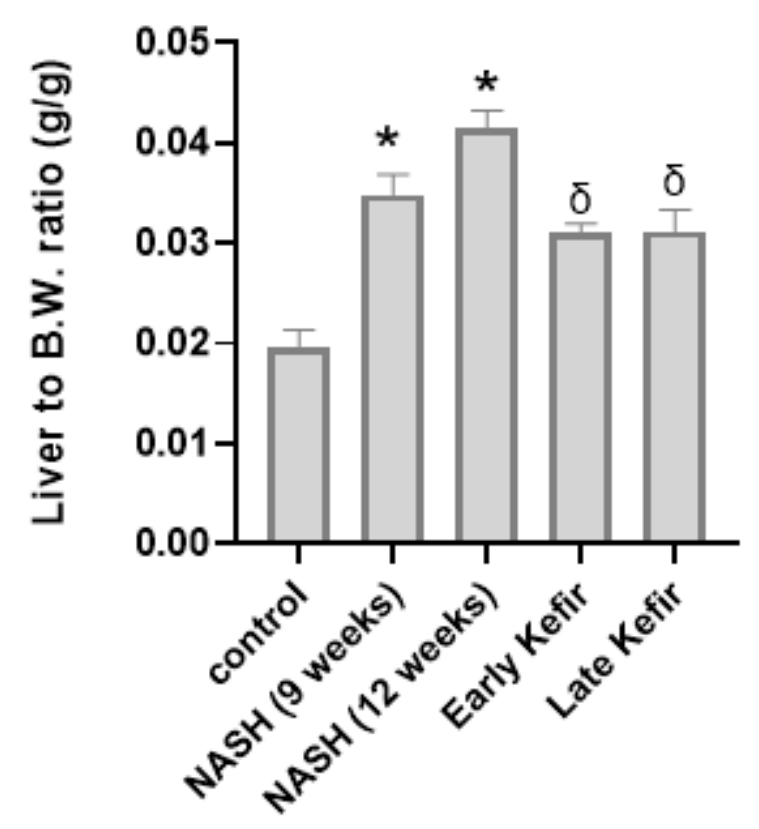

\section{Figure 2}

Liver/body ratio in different study groups. Values are mean \pm SEM; number of animals $=8$ rats/each group. One-Way ANOVA test followed by Tukey's multiple comparison test. * $P<0.001$ compared to control group, $\delta \mathrm{P}<0.001$ compared to NASH (12 weeks) group.

\section{Figure 3}

The effect of early and late Kefir treatment on hepatic steatosis, inflammation and fibrosis using HE and Masson's trichrome staining (Magnifications: $\times 100$ ). (A) Control group showed no pathological changes, (B) NASH (9 weeks) group developed mainly micro vesicular steatosis, mild hepatic lobular inflammation and mild fibrous septa(star) (C) NASH (12 weeks) group showed diffuse and extensive micro and macrovesicular steatosis, severe lobular inflammation, focal necrosis, hepatocellular ballooning, marked expansion of facultative hepatic progenitor cells (arrows), dense fibrous septa and collagen fibers (star) (D) Early Kefir and (E) Late Kefir groups showed a lower degree of steatosis, inflammation and fibrosis, where Early Kefir showed more remarkable histological improvements.

\section{Figure 4}

The effect of early and late Kefir treatment on hepatic steatosis, inflammation and fibrosis using HE and Masson's trichrome staining (Magnifications: $\times 100) .(A)$ Control group showed no pathological changes, (B) NASH (9 weeks) group developed mainly micro vesicular steatosis, mild hepatic lobular inflammation and mild fibrous septa(star) (C) NASH (12 weeks) group showed diffuse and extensive micro and macrovesicular steatosis, severe lobular inflammation, focal necrosis, hepatocellular ballooning, marked 
expansion of facultative hepatic progenitor cells (arrows), dense fibrous septa and collagen fibers (star) (D) Early Kefir and (E) Late Kefir groups showed a lower degree of steatosis, inflammation and fibrosis, where Early Kefir showed more remarkable histological improvements.
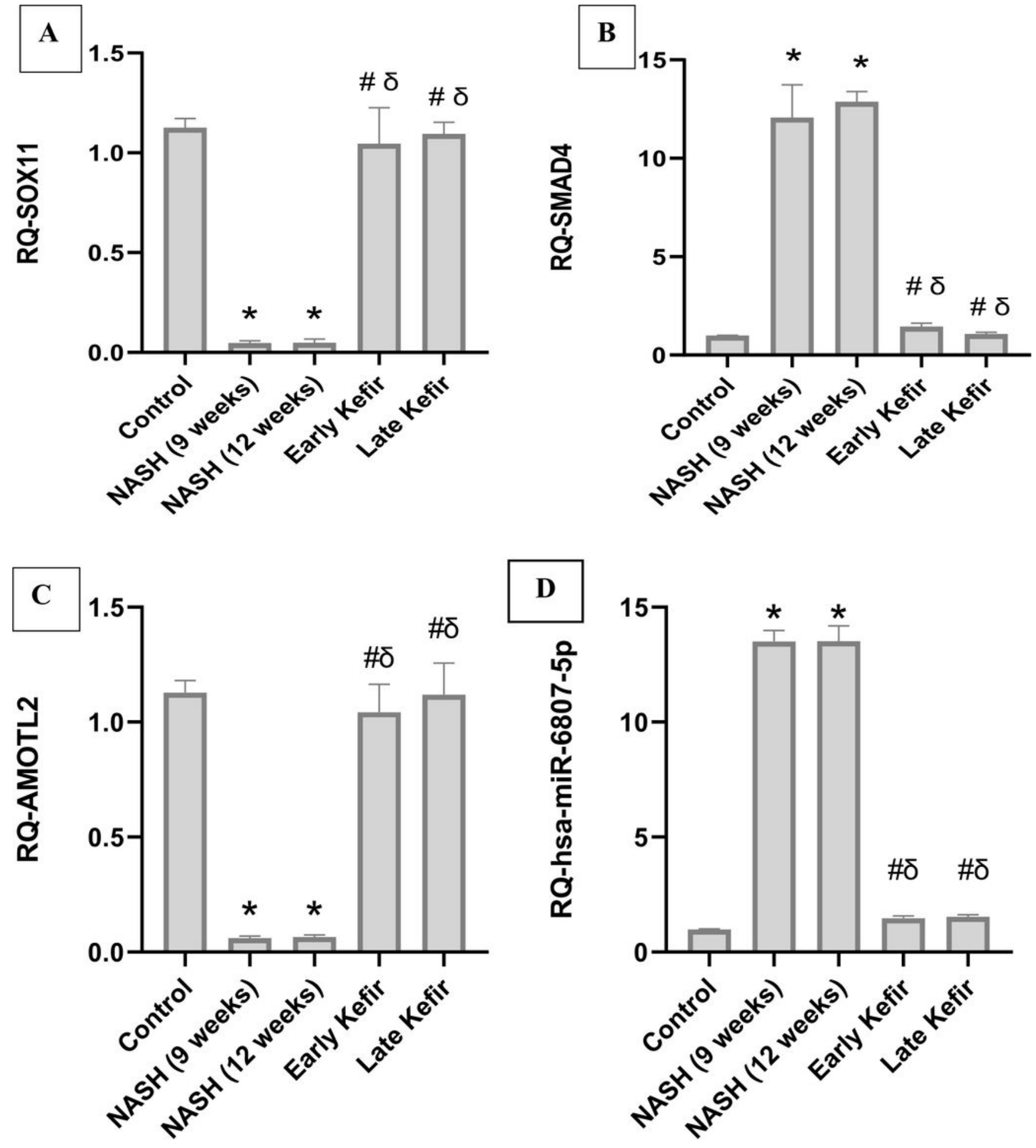

Figure 5

Effect of Kefir on the expression level of hepatic m-RNAs SOX11, SMAD4 and AMOTL2(A-C), hepatic miR $6807-5 p(D)$. Values are mean \pm SEM; number of animals $=8$ rats/each group. $* P<0.001$ compared to 
control group, \# P $<0.001$ compared to NASH (9 weeks) group, $\delta \mathrm{P}<0.001$ compared to NASH (12 weeks) group. One-way ANOVA followed by Tukey's multiple comparison test. RQ= relative quantification, SOX11 = SRY-RELATED HMG-BOX GENE 11, SMAD4= SMAD Family Member 4, AMOTL2=Angiomotin-like 2, hsa-miR 6807-5p= Homo sapiens miR 6807-5p.
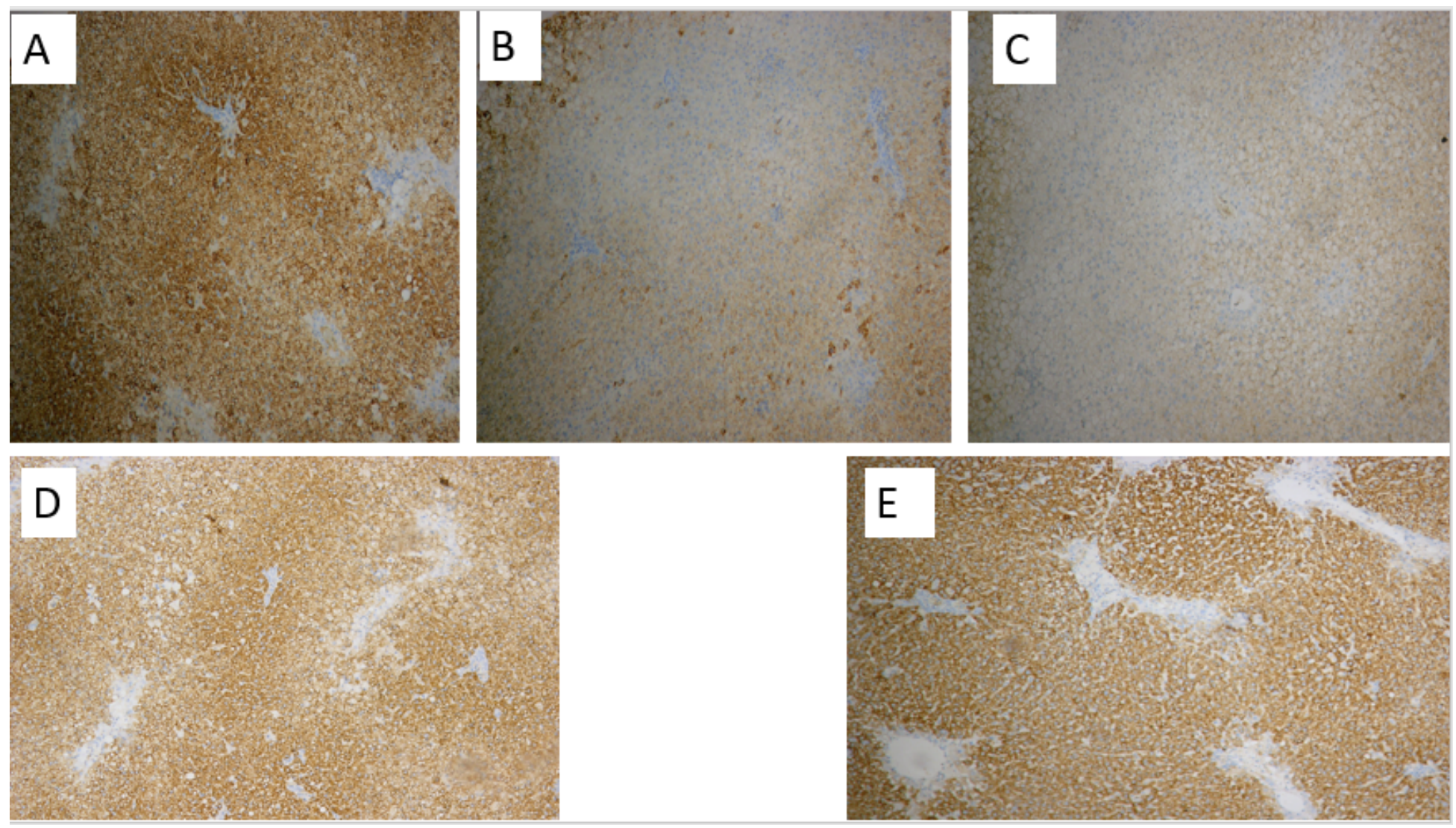

\section{Figure 6}

Immunohistochemical staining for hepatocyte specific antigen (HepPar1). (A) Controls showed a strong signal with Hep Par 1 (hepatocyte marker) producing distinct granular, cytoplasmic staining of hepatocytes, whereas in both (B) NASH (9 weeks) and (C) NASH (12 weeks) groups were mostly negative with HepPar-1. By comparison, (96\%) hepatocytes in (D) Early Kefir group were immunoreactive for HepPar-1 and (E) Late Kefir group tended to show weaker, patchy positivity (HepPar1, ×100) indicated to hepatocyte differentiation. 


\section{A}
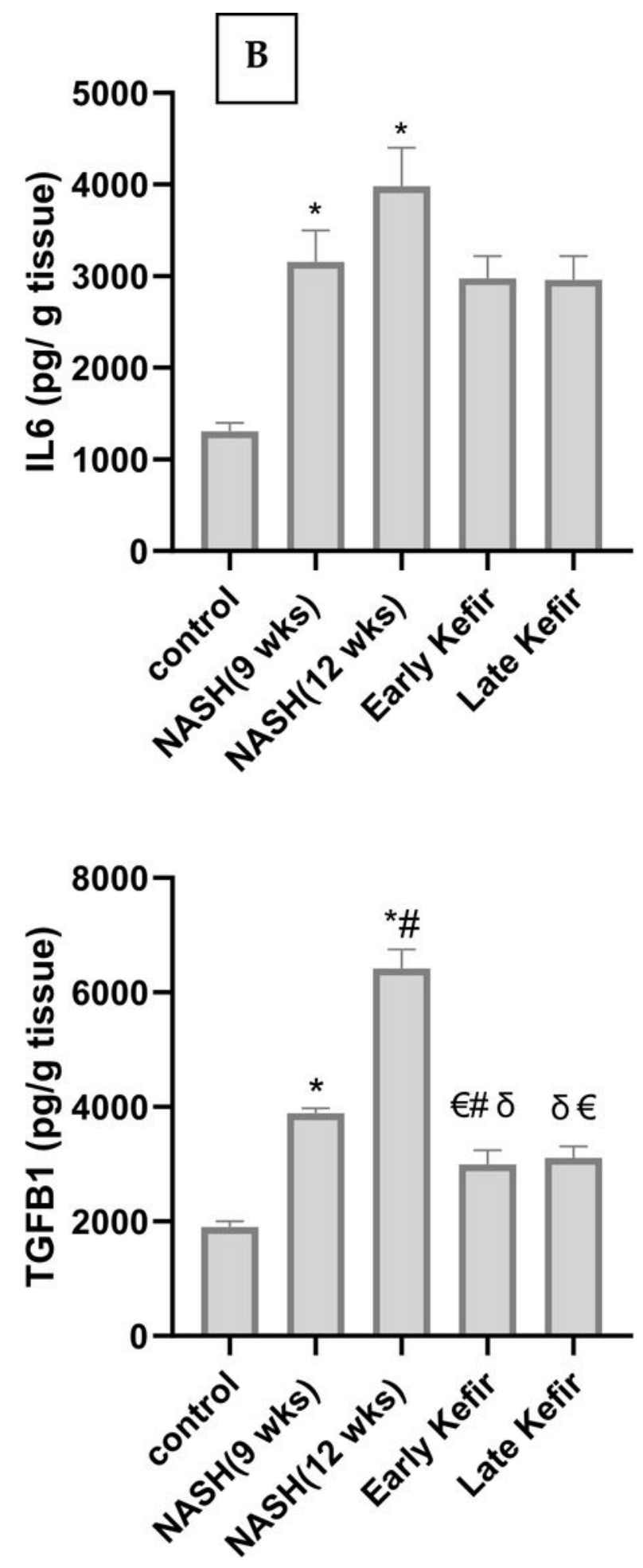

Figure 7

The effect of Kefir treatment on (A) Level of IL-6 (B) Level of TGF- $\beta 1$ in liver tissues from various groups. Values are mean $\pm S E M$, number of animals $=8$ rats/each group. ${ }^{*} P<0.001$ compared to control group, \# $\mathrm{P}<0.001$ compared to NASH (9 weeks) group, $\delta \mathrm{P}<0.001$ compared to NASH (12 weeks) group, $€ \mathrm{P}<$ 0.05. One-way ANOVA followed by Tukey's multiple comparison test. IL6= Interleukin 6, TGFB1= transforming growth factor beta 1 . 


\section{NAFLD/NASH}

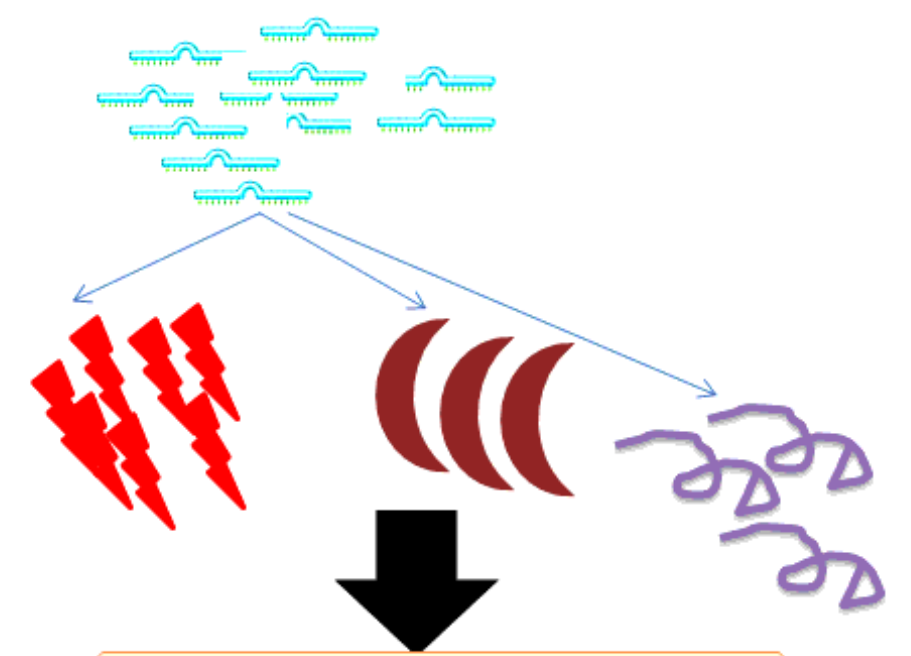

Deregulation of Hippo signaling and hepatocyte differentiation

(n)

Hsa-miR-6807-5p

Figure 8

Prove of concept map of the study hypothesis.

\section{Supplementary Files}

This is a list of supplementary files associated with this preprint. Click to download.

- supplimentaryclosed.docx 